\title{
Web Based Electronic Voting System Using Finger Print Authentication and GSM Module
}

\author{
Bilal Hussain Ch, Subayyal \\ Research Scholars, Department of Computer Science and Engineering, \\ University of Engineering \& Technology, RCET, Gujranwala Campus, Lahore, Pakistan
}

\begin{abstract}
Voting is the heart of a democratic country and it should be fair and square by all means .Every country in the world is spending a handsome amount on the electoral process to refine it to the next level. Instead of all the refinement on the electoral process there is always issues rises about the rigging in the elections. To counter this and to ameliorate the electoral process to next level we are proposing a system which includes a finger print scanner and GSM module. We have defined a methodology to counter all the hidden holes in the electoral process.
\end{abstract}

\section{Keyword: Finger print, GSM module}

\section{INTRODUCTION}

To completely understand our system first we have to understand the conventional voting system. In conventional voting system voters cast their vote using ballots papers. Which is a costly and troublesome process. The voters go to polling Booth to cast their vote and there are polling agents that guide the voters to cast their vote. The voters Than ticks their desired representative on the ballot paper. And this process is carried out for the others Voters as well. And at the end of the day the votes are counted. And the winner is selected with the most votes.

This process is difficult for the voters and as well as for the government.

Traditional voting process can be divided into different phases.

\section{Authentication:}

In this phase, voter authenticates himself or herself by showing his or her voting card, this step is public and verified by the presiding officer. At the end of authentication process, presiding officer give a ballot paper to voter to cast his or her vote.

\section{Vote:}

The vote takes place in a protected booth where voter cannot be seen by any person. The voter cast their vote by writing it with a pen on the paper ballot, folds the ballot paper and put into the ballot box where all the votes are mixed.

\section{Vote counting:}

At the end of voting time, the presiding officer collect the ballot box containing all ballot papers and submit it to the counting center. After that with the help of members of the election committee nominated by election commission of India, the ballot boxes are opened and votes are counted and the results are then announced.

\section{Verification:}

Various types of verification process are used, most procedure are public and verified by the representative of candidates of competing parties. Recount is also possible if there is any fraud or error.

In the $19^{\text {th }}$ century electronic voting system is also presented and it got implemented in most of the European countries and USA. In the E voting, voting machines are placed in the public areas so that the users can access them easily and cast their vote. It made the electoral process a whole lot faster but with the further inventions and research it was established that this voting process is not as safe as it is considered. Voting machines can be hacked and the results can be changed. 
To avoid all this confusion and trouble we have proposed a system that includes finger print scanning and GSM module.

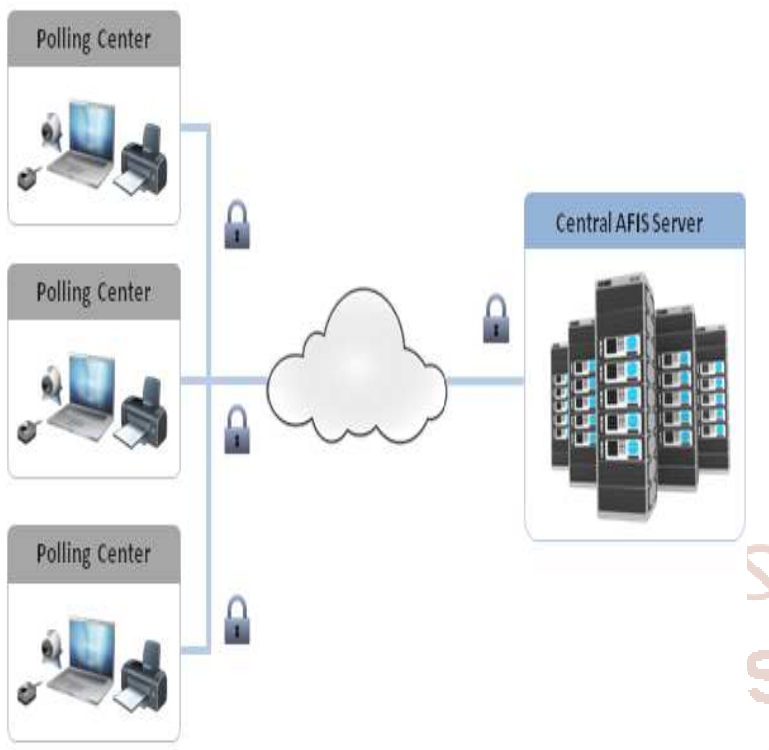

In our methodology there are three phases:

Registration phase: In this phase the voter will be registered and all the information regarding the voter is placed in the database. In this phase the voter will be prompted to input all his/her personal information in the required field like his ID card number, age , name, cell phone number. And after that the user will be asked to place the finger on the finger print device so that the image of the finger is placed in the database. After all this, registration phase will be completed.

\section{Authentication phase:}

This phase will start when the user is about to cast the vote. When the user go the website to cast the vote, before casting the vote this phase will begin. The user is asked to place the finger on the device, first the user is authenticated with the information saved in the database at the time of registration. If the user is authenticated the user will be given permission to cast the vote.

Bometric Enrciment (al earlier time')

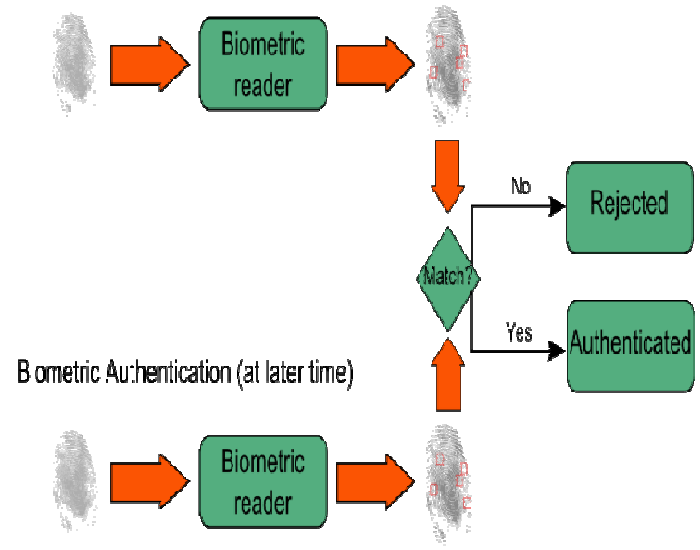

\section{Casting phase:}

In this phase the user will be given the privilege to cast the vote. If all the steps before are satisfied and completed then the user will be promoted to this phase.

In this phase the user cast the vote .And at the end the result will be calculated.

\section{Benefits of our methodology:}

The benefits of our methodology are:

\section{$>$ Fairness:}

Our system will introduce fairness in the electoral process to next level. As the methodology proposed by us is nearly impossible to break. So it will introduce fairness in the electoral process.

\section{Eligibility of voters:-}

In our methodology we have made sure that only authorized will be able to cast the vote. Unauthorized voters will be caught at the start of the process and will not be allowed to cast the vote.

\section{Uniqueness:-}

We have defined uniqueness in our system. Every voter has the unique identity because of the unique finger print. No voter would be allowed to cast the vote more than one time.

\section{Privacy:-}

We have paid special attention to privacy in our system. As the user will cast the vote over the internet and can cast the vote anywhere.

So no one can access the information regarding the vote of the voter. His/her privacy will be maintained.

\section{$>$ Accuracy:-}

Our methodology is accurate. We have introduced accurate and dependable devices in the system. They will provide the most accurate result is all conditions.

\section{Efficiency:-}

Efficiency is the important factor that we have considered.

Our system will enhance the efficiency to the next level. Our methodology will provide the result in minimal time and with precision. 
Difference between other e-voting systems and our methodology:-

Due to intense need of the fairness in the electoral process various modules have been proposed. Various systems have been implemented in which finger print authentication is using. But there is a main difference between our methodology and other e voting scheme.

First of all our methodology includes GSM modules that is absent in other types of systems. And besides that we are using Finger print authentication modules, functions of which are created in MATLAB and advanced acquisition and comparison techniques are used.

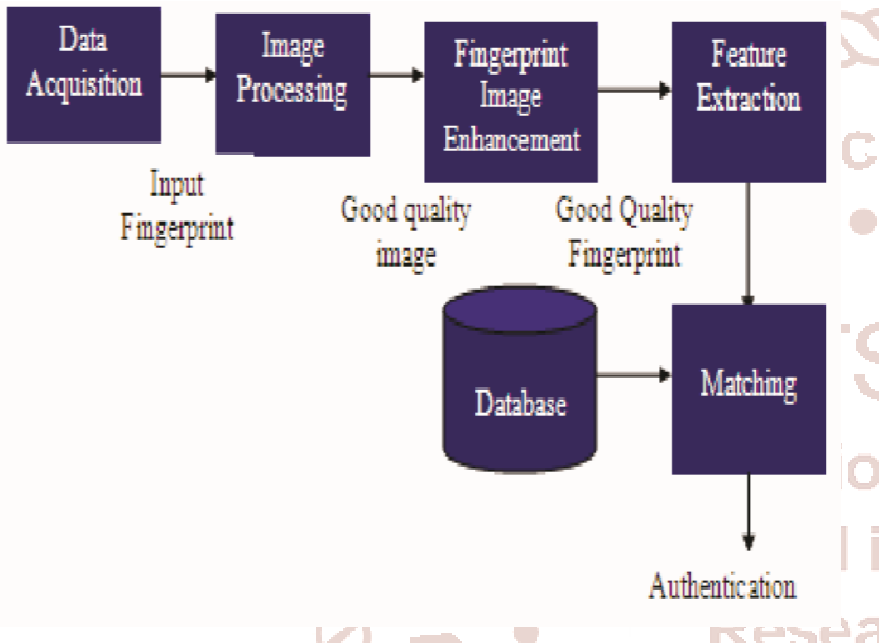

We have used various built in functions and also created some functions in MATLAB to enhance the quality of the input image and to store it in the database.

The use of the GSM module is that when the user authorizes itself for the casting of the vote the message is send to the given number of the voter and the message contains the code for the voting. The user is asked to input the code in the required field in order to cast the vote.

\section{Explained view of the system:-}

We are using two basic modules GSM and the Finger print scanner. The use of both devices is immense and vital.

Our proposed methodology is client/server based and web based. The voter will cast the vote using Internet.

Our methodology consists of 3 phases:-

\section{Registration phase:-}

In this phase the voter will be registered. This is the first phase in the system. The user will login in to the system and opens the website to register itself. The user will be prompted to give the basic information regarding himself / herself.

Remember our methodology is client/server based. The voter will be the client. And its web based architecture. After the user will give all the information about himself / herself then user will be asked to provide the cell phone number and after that the user is asked to place the finger on the device so that the system can take the fair good impression of the finger. We have used some MATLAB functions and have created some of the functions regarding image acquisition and image enhancement to get a clear impression of the finger.

After all the working the voter will be registered and a unique voter id will be generated in database for the user. And thus the first phase will be completed this way.

\section{Authentication phase:-}

In this phase the voter will be authenticated as it name specifies.

This phase will start right before the voting and the last phase. In this phase, when the voter is ready to cast the vote the voter will be prompted to place the finger on the device so that the system can take a fair good impression of the thumb. The image obtained will be compared with the image placed in the database for that voter. If the image matches with the one in the database the user will be authorizes and asked to cast the vote. And if the image doesn't matches with the one on the database the voter is not authorized and will be not asked to cast the vote.

We have used some of the MATLAB functions and also created some to remove the ambiguity of matching the finger prints.

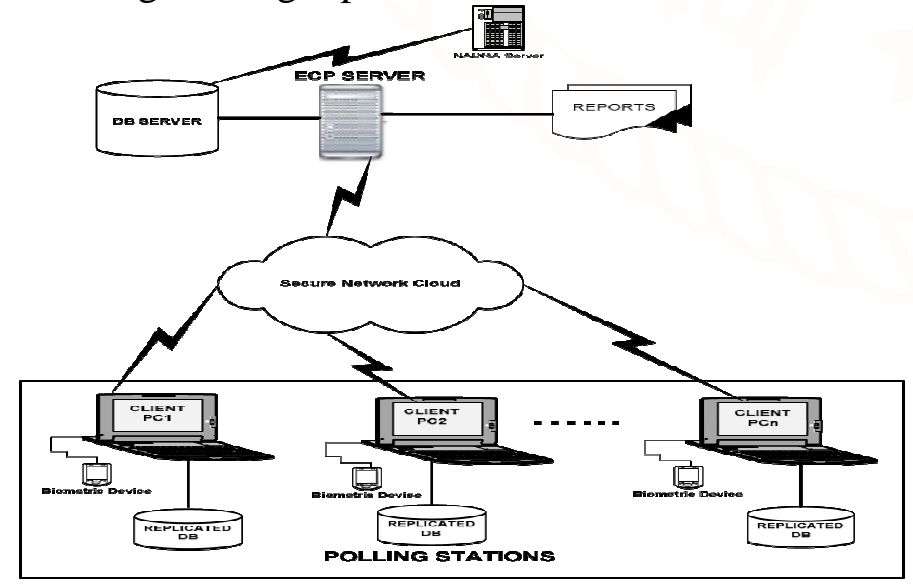




\section{Casting phase:-}

After all the registration and authentication the voter will be finally prompted to cast the vote.

The list of candidates will be visible to the voter and the voter can tick the right one he/she wants.

After the casting it is very easy and feasible to count the votes. The votes would automatically be counted at the end because of the efficiency of the system. And the winner will be announced.

Flowchart:-

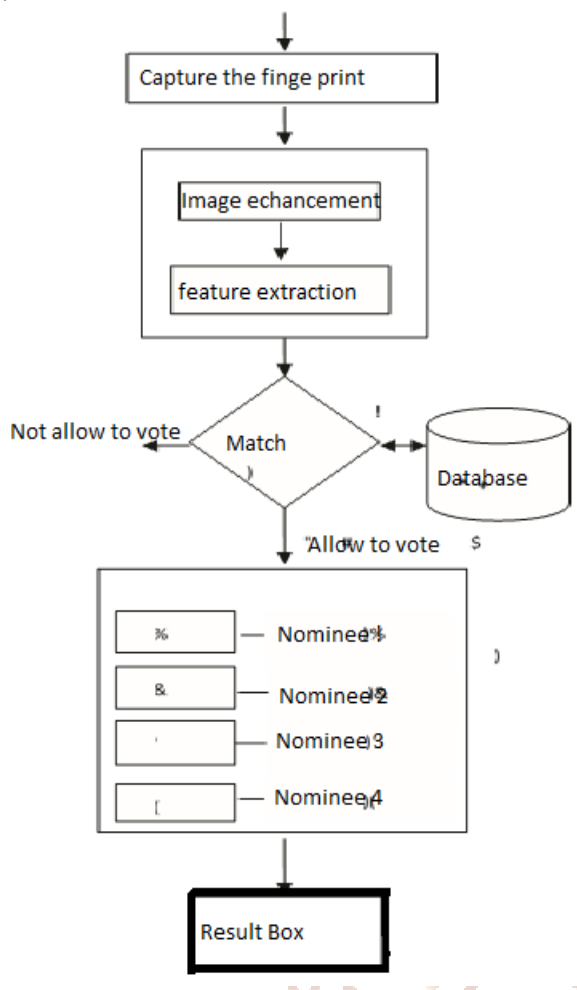

\section{Conclusion:-}

We have defined the methodology using two modules Finger print scanner and the GSM module. Both the modules have vital importance in the system. Our methodology is that we are using a client/ server based architecture. In which the voter is a client. It's a web based architecture. First the user is registered with his/her unique finger print in the database of the government. After the registration phase there comes the authentication phase and in the end there is casting phase. In the authentication phase finger print of the voter is compared with the finger print saved in the database at the time of registration. . The use of the GSM module is that when the user is about to cast the vote a code is send to the cell phone of the voter. The user will be asked to place the code in the field given to cast the vote. Our methodology is unique, fair, adaptable, secure and efficient.

\section{Future work:-}

In future the voting process can be more verified and enhanced using our methodology.

We can attach other modules as well to enhance the efficiency of the system. We can attach iris detection and face detection to maximize the efficiency.

We can also attach speech recognition module.

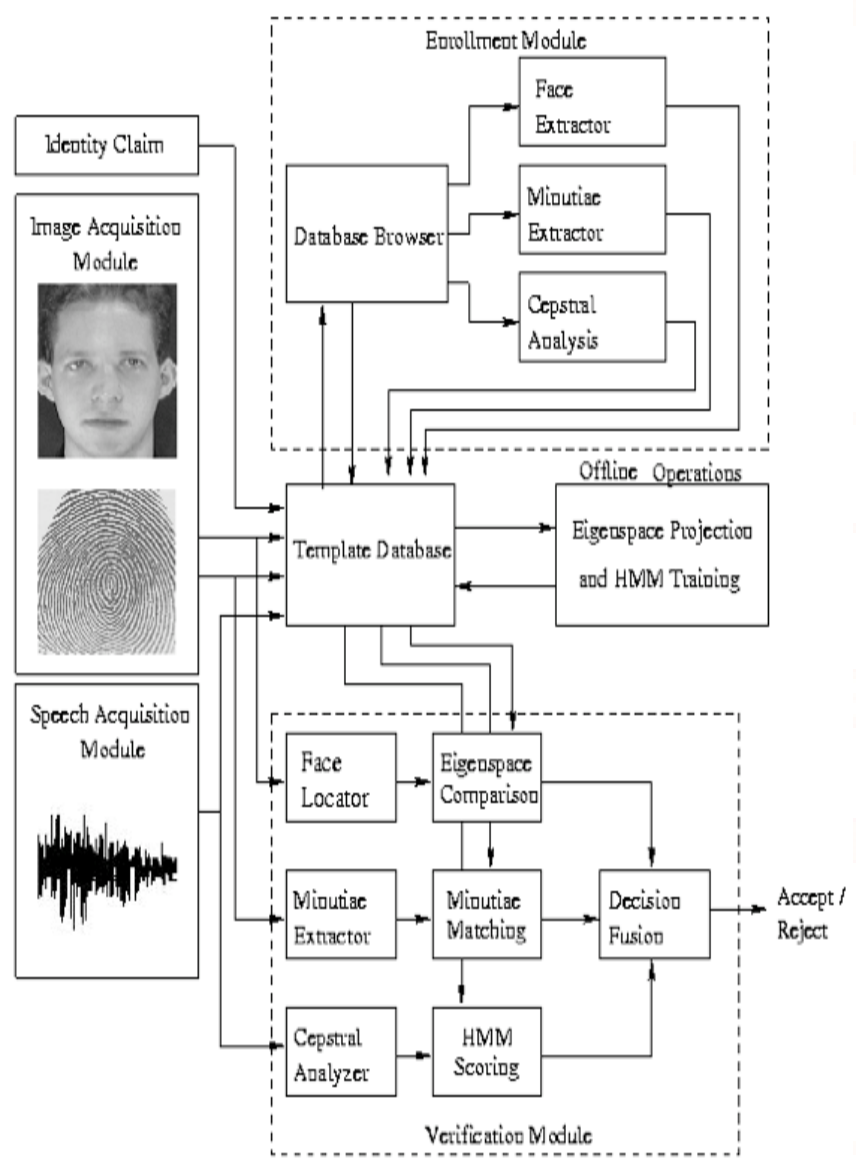

References:-

1. R. Mercuri, "Electronic Vote Tabulation Checks and Balances", $\mathrm{PhD}$ thesis, University of Pennsylvania, Philadelphia, PA, October 2000.

2. D. Rubin, "Security considerations for remote electronic voting. Communications of the ACM, 45(12):39-44", December 2002. http://avirubin.com/e-voting.security.html

3. T. E. Carroll, D. Grosu, "A Secure and Efficient Voter-controlled Anonymous Election Scheme," International Conference on Information Technology: Coding and computing, as Vegas, pp.721 - 726, 2005.

4. C. H. Chen, C. M. Lan, and G. Homg, "A Practical Voting Systems for Small Scale Election," 3rd International Conference on 
Information Technology: Research and Education, Hsinchu, pp.322 - 326, 2005.

5. Z. Claudia, R. H. Francisco, and O. A. Daniel, "SELES: An e-Voting System for Medium Scale Online Elections," Process of the Sixth Mexican International Conference on Computer Science, Puebla, pp.50-57, 2005.
6. Maltoni D, Maio D, Jain A K, Prabhakar S, Handbook of Fingerprint Recognition, 2nd ed., Springer, 2009

7. Altun A A, Kocer H E, Allahverdi, "Genetic algorithm based feature selection level fusion using fingerprint and iris biometrics", Int. J. Pattern Recog. Artif. Intell. (IJPRAI), 22(3): 585600, November 2008.

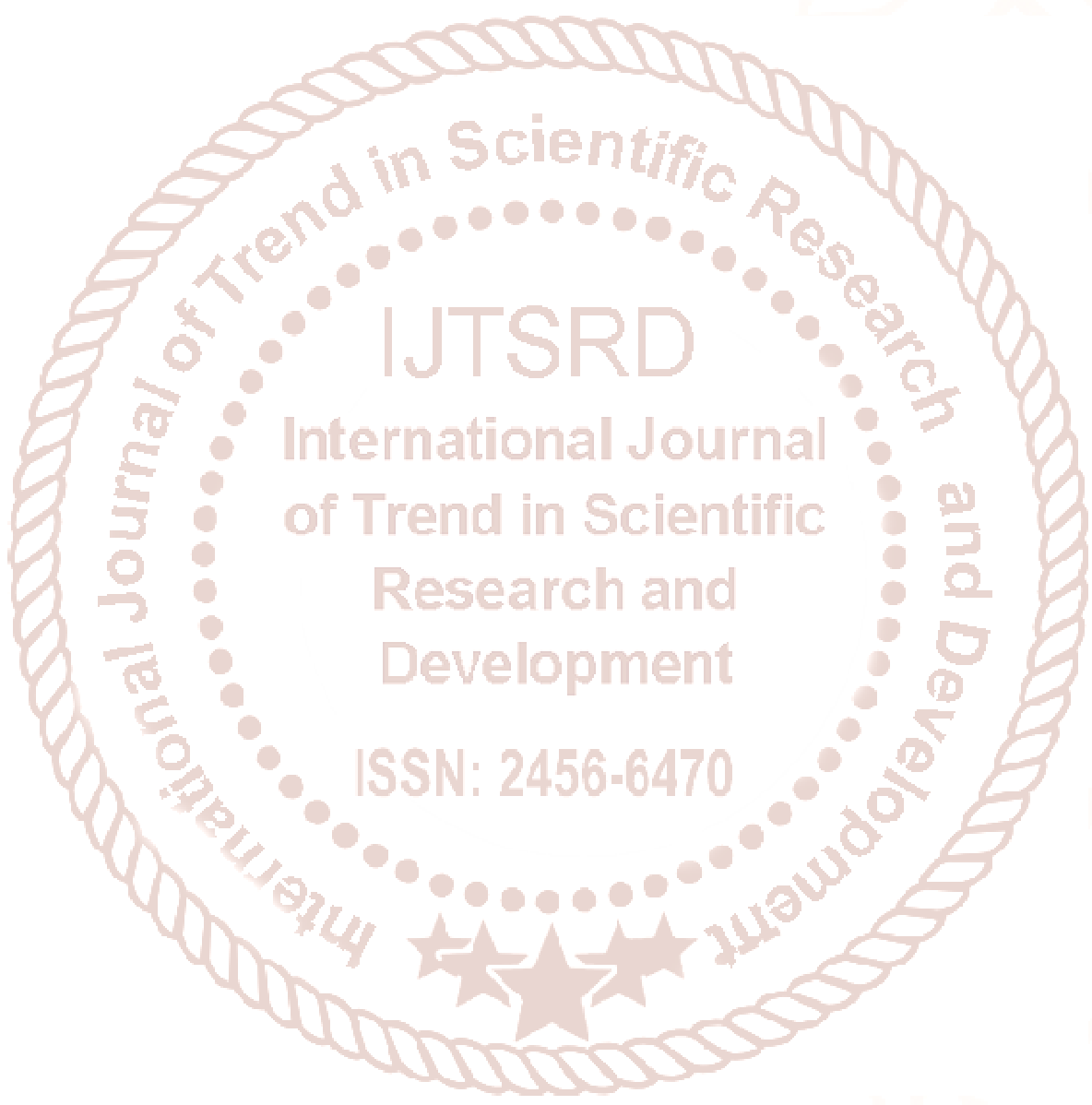

